\title{
Band gap formation in thin plates with a periodic array of resonators
}

\author{
Sahar Zouari ${ }^{1, a}$, Jean-Michel Genevaux ${ }^{2}$, Julien Brocail ${ }^{1}$ and Frédéric Ablitzer ${ }^{2}$ \\ 1 ESTACA'LAB, Pôle Mécanique des Matériaux Composites et Environnement, ESTACA-Campus Ouest, \\ Rue Georges Charpak, BP, Laval, France \\ 2 LAUM, Université du Maine, CNRS UMR 6613, LAUM, Avenue Olivier Messiaen, Le Mans, France
}

Received 2 December 2015, Accepted 27 October 2016

\begin{abstract}
A resonator assembly consisting of a two-dimensional periodic array (mass-screws) mounted to a thin homogenous plate was used to investigate the vibration characteristics of locally resonant (LR) phononic plates. The numeric simulations employed the finite element method to calculate the band structures of the proposed periodic plates and to analyze the effect of geometry parameters on the evolution of the flexural band gap behavior. To experimentally validate the predictions for these theoretical examinations, two measurements with the LR phononic plates were obtained with respective lattice constants $a=40$ and $50 \mathrm{~mm}$. The tested plate was clamped on one side to a shaking table to generate a plane wave, propagating in the Ox-direction. Obtained experimental measurements of the wave attenuation in this direction are in good agreement with the theoretical frequency of both complete and directional band gaps.
\end{abstract}

Key words: Local resonators / phononic plate / band gap / frequency response / vibration

\section{Introduction}

Phononic crystals are periodic materials with identical unit cells that are able to act as mechanical filters of wave propagation. The periodic effect causes an attenuated wave propagation in a given frequency range [1,2], called a band gap. There are two main methods to generate band gaps. The first approach is based on the Bragg scattering mechanism, whose wavelengths are on the scale of the structure's periodicity. The second approach is the LR mechanism, which utilizes the resonant modes of the microstructures in each unit cell. The LR mechanism was first introduced by Liu et al. [3], who proposed a LR phononic crystal composed of a periodic array of coated lead balls immersed in an epoxy matrix. Their study found LR band gaps that were two orders of magnitude lower than the Bragg gaps. Xiao et al. [4] proposed a LR phononic plate composed of a two-dimensional periodic array of spring-mass resonators mounted to a thin homogeneous plate. This structure represents a numeric model for understanding the basic wave propagation physics of LR phononic plates. Oudich et al. [5] demonstrated numerically and experimentally the existence of a lowfrequency LR band gap created by the local low-frequency resonance of soft rubber pillars coupled with the plate's

${ }^{a}$ Corresponding author: sahar.zouari@estaca.fr modes. A similar idea was considered by Yu et al. [6], who studied a rubber matrix plate with periodic steel stubs. Recently, Li et al. [7] showed that introducing a composite plate-type acoustic metamaterial, constituted of one-side cylindrical stubs deposited on a two-dimensional binary locally resonant phononic plate, causes the local resonance band gaps to shift to a lower frequency. This method makes it possible to obtain a relative bandwidth that is significantly enlarged (by a factor of three) compared with one-sided LR stubbed plates. Hu et al. [8] studied a similar LR phononic structure made by depositing the heavy cylinder LR stubs squarely onto both sides of a thin elastic composite plate. Nouh et al. [9] examined an LR plate system consisting of a base structure composed of cavities filled by a viscoelastic membrane that supported a small mass constituting the source of local resonance.

The present paper proposes a two-dimensional (2D) LR phononic plate composed of a thin homogeneous plate with an attached periodic array of resonators (massscrews). This periodic structure allowed us to easily modify various geometric experimental parameters. The present study focused on the resonant frequency of the resonator and periodicity variations, and its impact on the flexural wave band gap behavior of the proposed periodic structure. A finite element method (FEM) was used 


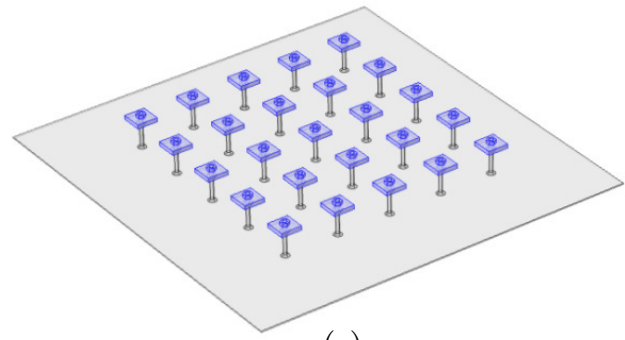

(a)

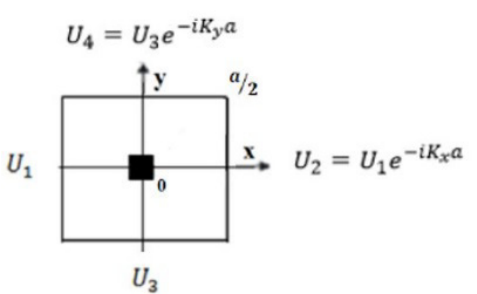

(b)

Fig. 1. (a) Configuration of the locally resonant phononic plate with periodically attached resonators (mass-screws). (b) Periodic boundary conditions for a square unit cell.

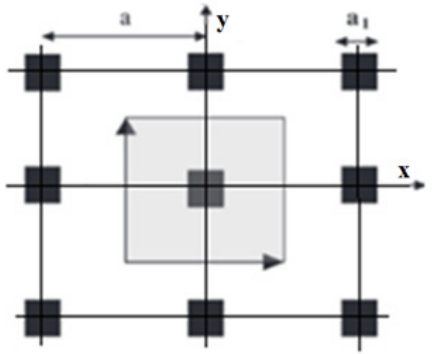

(a)

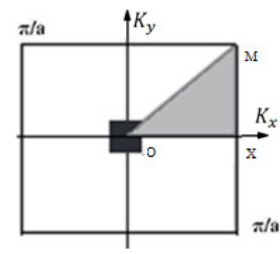

(b)
Fig. 2. (a) Cross section of a square unit cell. (b) First Brillouin zone of a square unit cell showing the irreducible region of the Brillouin zone (gray area).

to investigate wave propagation in these LR plates. Band structures were calculated to predict band gaps characteristics. To experimentally validate our theoretical predictions, we used different lattice constants to measure the unidirectional excitation response of the LR phononic plates.

\section{Model and method}

The studied LR phononic plate consisted of a periodic array of resonators (mass-screws) mounted to a thin homogeneous plate (Fig. 1a). Given the structure's periodicity, only one-unit cell was considered in the calculation. To obtain the band structure of the infinite periodic plate, the dispersion relation between the frequency and the wave number of a unit cell must be determined by varying the wave vector in the first irreducible Brillouin zone (Fig. 2). Bloch-Floquet periodic boundary conditions were used for the edges of the unit cell (Fig. 1b):

$$
U_{\text {destination }}=U_{\text {source }} e^{-i K_{F} a},
$$

where $U_{i}$ is the displacement vector, $a$ is the width of the unit cell, and the real number $K_{F}$ corresponds to the wave vector.

Using the Comsol 5.0 software suite with a finite element method, we calculated a series of dispersion relations to investigate the propagation characteristics of elastic waves in the proposed LR plate. Free tetrahedral elements quadratic interpolation functions were used to mesh the mass, screw, and bolts. The plate surface was meshed with the free triangular elements. By distributing of three elements, swept meshing was applied in the direction of the thickness of the plate. The efficiency of the finite element method for obtaining phononic crystal dispersion curves has been proven in previous studies $[7,10]$. The governing field equations for elastic wave propagation in solids are given by

$$
\rho \frac{\partial^{2} U_{i}}{\partial t^{2}}=\sum_{j=1}^{3} \frac{\partial}{\partial x_{j}}\left(\sum_{l=1}^{3} \sum_{k=1}^{3} C_{i j k l} \frac{\partial U_{k}}{\partial x_{l}}\right),(i=1,2,3)
$$

where $\rho$ is the mass density, $U_{i}(t, x, y)=U_{i}(x, y) e^{i \omega t}$ is the displacement, $t$ is the time, $C_{i j k l}$ is the elastic constant, and $x_{j}(j=1,2,3)$ represents the coordinate variables $x, y$, and $z$, respectively. By using these equations with the Bloch-Floquet periodic boundary conditions, we can calculate the dispersion relations for the proposed structures.

Three examples of LR plate systems were analyzed using the same plate, lattice constant, and added mass. The geometrical parameters were chosen as follows: a lattice constant of $a=40 \mathrm{~mm}$, a plate thickness of $e=1$ $\mathrm{mm}$, and a width of $a_{1}=15 \mathrm{~mm}$ and a thickness of $e_{1}=3 \mathrm{~mm}$ for the square steel filler The square fillers were made of steel (Young's modulus $E=205 \mathrm{GPa}$, density $\rho=7850 \mathrm{~kg} . \mathrm{m}^{-3}$, Poisson's ratio $\left.\nu=0.28\right)$. The thin host plate was made of aluminum $(E=70 \mathrm{GPa}$, $\left.\rho=2700 \mathrm{~kg} \cdot \mathrm{m}^{-3}, \nu=0.33\right)$. The screw was made of nylon $\left(E=2 \mathrm{GPa}, \rho=1150 \mathrm{~kg} \cdot \mathrm{m}^{-3}, \nu=0.4\right)$. As shown schematically in Figure 3, the square filler was threaded between two hex nuts and mounted either to the top (Fig. 3a) or the middle (Fig. 3b) of an M3 $\times 40$ screw or to the top of an M3 $\times 25$ screw (Fig. 3c).

The LR plate was composed of repetitive unit cells. This periodic nature of the plate caused it to act as a mechanical filter for wave propagation. Figures $4 a-4 c$ shows the calculated dispersion relations of the proposed LR plates with the three unit cells (described in Figs. 3a-3c). The vertical axis represents the frequency $[\mathrm{Hz}]$, and the horizontal axis is the reduced wave vector taken along the first irreducible Brillouin zone MOXM (Fig. 2b). According to Bloch's theorem, these measurements are sufficient to determine the complete and directional frequency band gaps, provided the group velocity of the points $v_{g}=\nabla_{k} \omega$ 


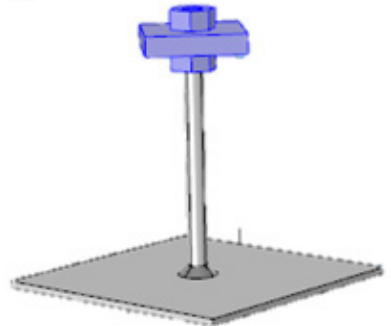

(a)

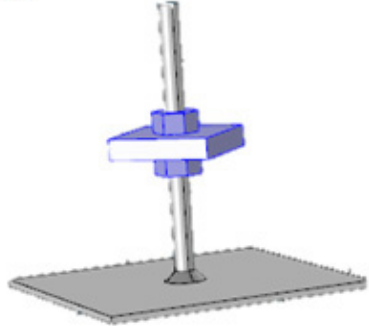

(b)

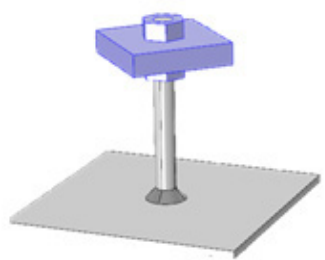

(c)

Fig. 3. Three resonator (mass-screw) configurations: (a) M3 $\times 40$ screw with top-mounted mass, (b) M3 $\times 40$ screw with center-mounted mass, and (c) M3 $\times 25$ screw with top-mounted mass.

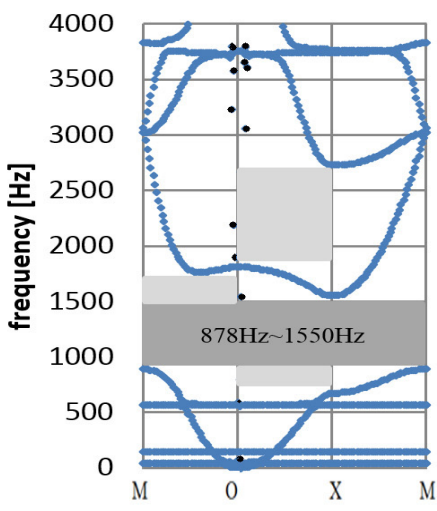

(a)

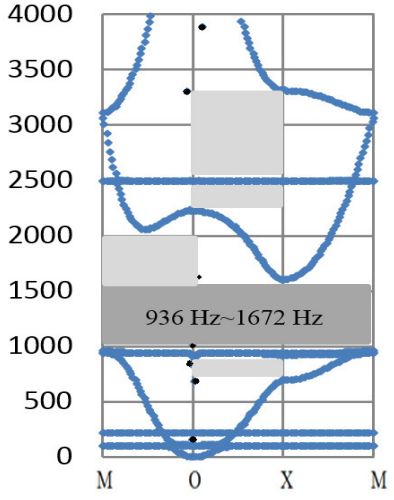

(b)

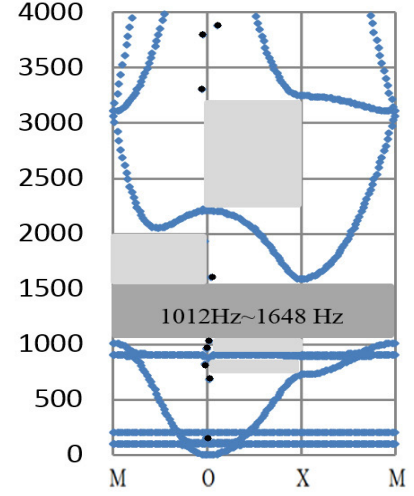

(c)

Fig. 4. Band structures of the three LR configurations: (a) M3 $\times 40$ screw with top-mounted mass, (b) M3 $\times 40$ screw with center-mounted mass, and (c) M3 $\times 25$ screw with top-mounted mass.

does not have an infinite magnitude [11]. Although we focused exclusively on the flexural band gap, the band structures also showed high group velocity modes (black points in Fig. 4), which correspond to the compression or shear mode of the plate. The horizontal curves correspond to the resonant modes, for which energy was localized mainly in the resonators (mass-screws): plate deformation was negligible compared with the deformation of the resonators. The frequencies of the horizontal curves were close to the resonance frequencies of the uncoupled resonators clamped to the base.

The band structures show the existence of complete and directional frequency band gaps for the three configurations (highlighted by the gray areas in Fig. 4). The complete band gaps had similar frequency widths. Thus, varying the resonator's stiffness (represented by changing the positions of the mass on the screws) had a negligible influence on the frequency width of the band gap. To better understand the evolution of the band gap, Figure 5 shows the bare aluminum plate band structure (dotted green lines) and the frequency resonances of the local resonator (horizontal dashed red line). The Figure 5 shows that the horizontal line of the local resonator disappears near a frequency of $1900 \mathrm{~Hz}$ : a strong coupling with the plates gives the complete band gap of between 1000 and $1650 \mathrm{~Hz}$. Moreover, the upper complete band gap edge is determined by the Bragg conditions in the

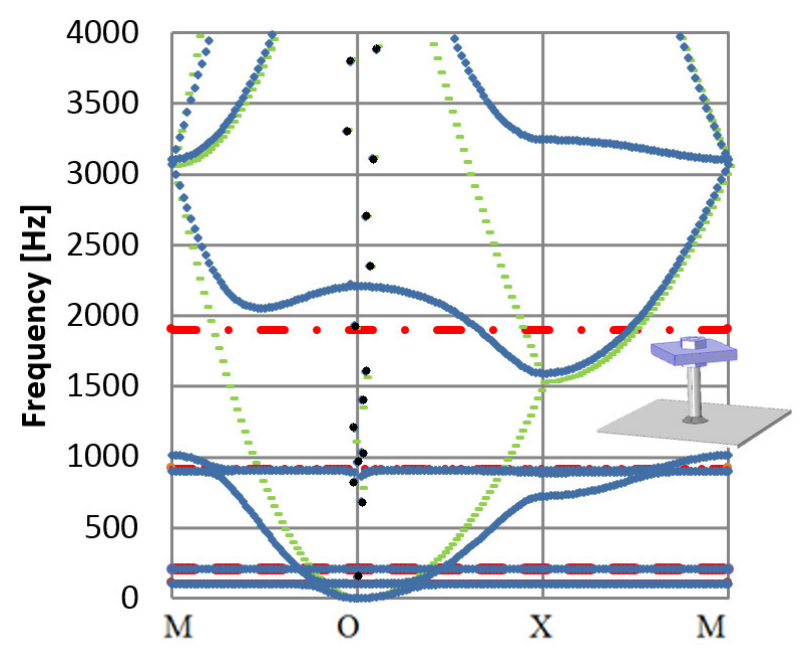

Fig. 5. Band structures of: the LR aluminum plate with a periodic resonator $\mathrm{M} 3 \times 25$ screw with top-mounted mass (blue line), M3 $\times 25$ screw with top-mounted mass (dashed red line), and bare aluminum plate (dotted green line).

Ox-direction ( $a=\frac{\lambda_{p}}{2}$, where $\lambda_{p}$ represents the flexural wavelength of the thin plate), which gives the Bragg frequency $f_{B}=\frac{1}{2 \pi}\left(\frac{\pi}{a}\right)^{2} \sqrt{\frac{D}{\rho h}}[4]$. Thus, the Bragg and res- 


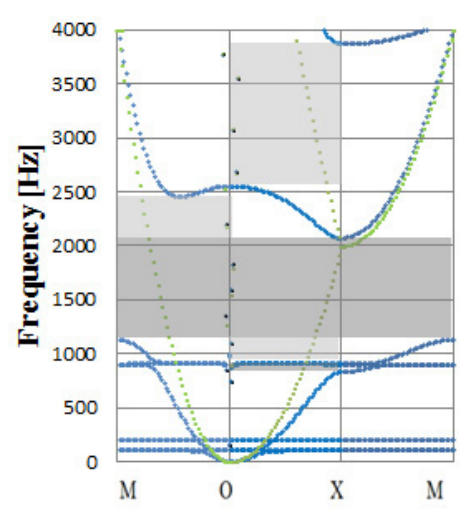

(a)

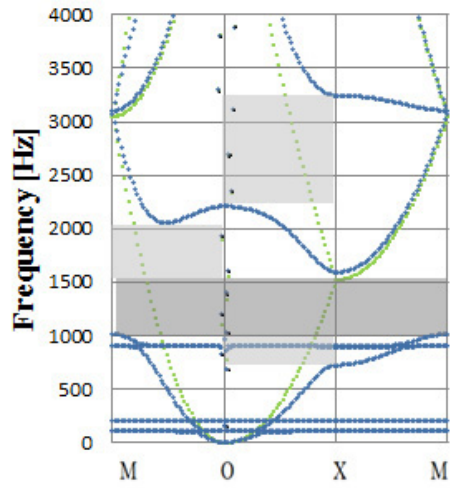

(b)

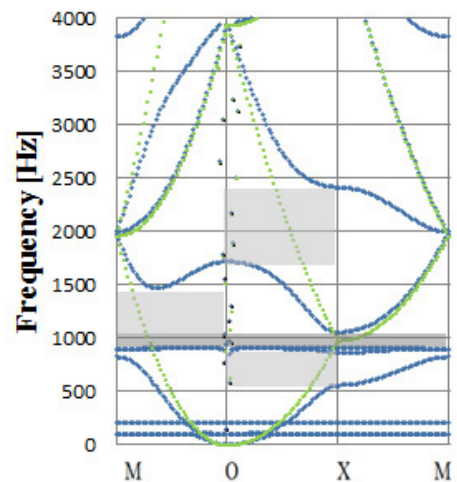

(c)

Fig. 6. Band structures of bare aluminum plate (dotted green line) and band structures of the LR assembly consisting of a thin aluminum plate and an M3 $\times 25$ screw with a top-mounted mass (blue line): (a) $a=35 \mathrm{~mm}$, (b) $a=40 \mathrm{~mm}$, and (c) $a=50 \mathrm{~mm}$.

onance band gaps are joined in the band structures to create a complete band gap with a great width.

To illustrate the mechanism responsible for the band gap position in the LR plate, we investigated the effect of the lattice constant on the band gap. Figures $6 \mathrm{a}-6 \mathrm{c}$ shows the band structures of the LR plate consisting of an aluminum plate with a periodic mass mounted to the top of the M3 $\times 25$ screw for lattice constants of $a=35$ $\mathrm{mm}, 40 \mathrm{~mm}$, and $50 \mathrm{~mm}$. The greater the lattice constant $a$, the lower the upper edge of the band gap and the thinner the complete band gap. The position of the complete band gap changed because its upper gap edge was always located at the Bragg frequency. Thus, for such a Bragg band gap, the position of the first directional band gap in the $O x$-direction is in accordance with the complete band gaps (Fig. 6). However, in the same direction, the position of the second directional band gap can be changed significantly by tuning the resonant frequency of the resonator (as shown in Fig. 4), suggesting that it behaves similar to a resonance band gap.

\section{Experimental results}

To validate the two numerical approaches presented in Section 2, the experimental analysis was based on the resonator configuration described in Figure 3c. The LR phononic plates consisted of a $600 \mathrm{~mm}$ long $(x-$ direction), $500 \mathrm{~mm}$ wide ( $y$-direction), and $1 \mathrm{~mm}$ thick $(z$ direction) aluminum base plate. The plate was attached to a square array of $11 \times 6$ mass-screw resonators (as shown in Fig. 10a). The resonator array had a lattice constant of $a=40 \mathrm{~mm}$. The behavior of the LR plate assembly was compared with that of a bare aluminum reference plate of identical length. To generate a plane wave propagating in the $x$-direction, the plates were each clamped on one side to a shaking table (Ling Dynamic Systems: LDS V850-440-SPA32K) whose displacement was in the $Z$-direction (Fig. 7). The other sides of the plates were left free. The imposed displacement along the clamped side of the plate consisted of white noise with a frequency range of between 20 and $2000 \mathrm{~Hz}$ and an amplitude of $0.011 \mathrm{~mm}$ RMS. This excitation is equivalent to an inertial uniform surface load of the structure. Two accelerometers (PCB M352C66) were used to measure the excitation acceleration: one accelerometer was placed on the shaking table; the other was located on the rigid beam clamping the plate (Fig. 7). The out-of-plane response was measured with a Scanning Laser Doppler Vibrometer (SLDV, Polytec PSV-400); the measuring laser beam swept over the surface without resonators. The measurements were taken by scanning $11 \times 13$ points over a $0.04 \mathrm{~m}$ $\times 0.04 \mathrm{~m}$ (Fig. 10b). For each position 20 averages were used to record the fast Fourier transform (FFT). A resolution of $625 \mathrm{MHz}$ with $0 \%$ overlap and a Hamming window function was used to determine the frequency response.

The curves shown in Figure 8 contrast the excitations measured on the shaking table with those measured near the clamp of the plate. Possibly due to local resonance, measurements taken near the clamp varied with frequency, which suggests that the clamping was flawed. The acceleration of the table (input1) is used in the following.

Through an FFT, the SLDV achieved the frequency response function (FRF) of each scan point $\left(H_{1}(f)\right.$ : acceration ${ }_{V i b} /$ acceleration $\left._{\text {Ref }}\right)$. The following equation calculates the average of $H_{1}$ for each line of the measured scanning points in the $O x$-direction:

$$
H_{\text {line }(j)}=\frac{1}{n} \sum_{i=1}^{n} \text { magnitude }\left(H_{1}(i j)\right) ; j=\{1,2,3,4,5\}
$$

Figures 9 and 10 show measured averaged frequency response $H_{l i n e(j)}$, represented by curves, according to frequency for the first five detection lines of the LR plate (Fig. 9) and of the reference plate (Fig. 10). Figure 9 shows a significant decrease in $H_{\text {line }(j)}$ for values of $j=1$ to 5 between 1000 and $1650 \mathrm{~Hz}$ (gray area). The vibration attenuation increased as a function of the position of the measured line over the excited side. This decrease in vibration is absent in Figure 10, which corresponds to the bare plate case. We thereby experimentally demonstrated 
S. Zouari et al.: Mechanics \& Industry 18, 304 (2017)

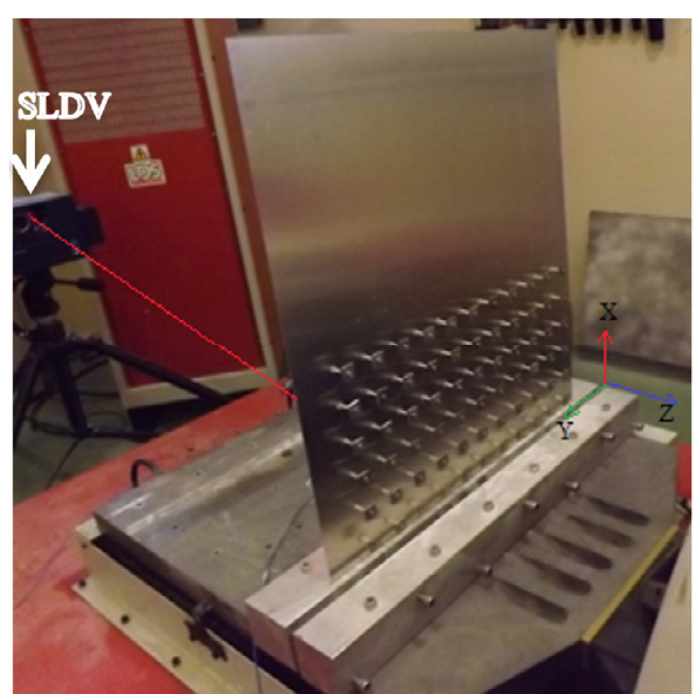

(a)

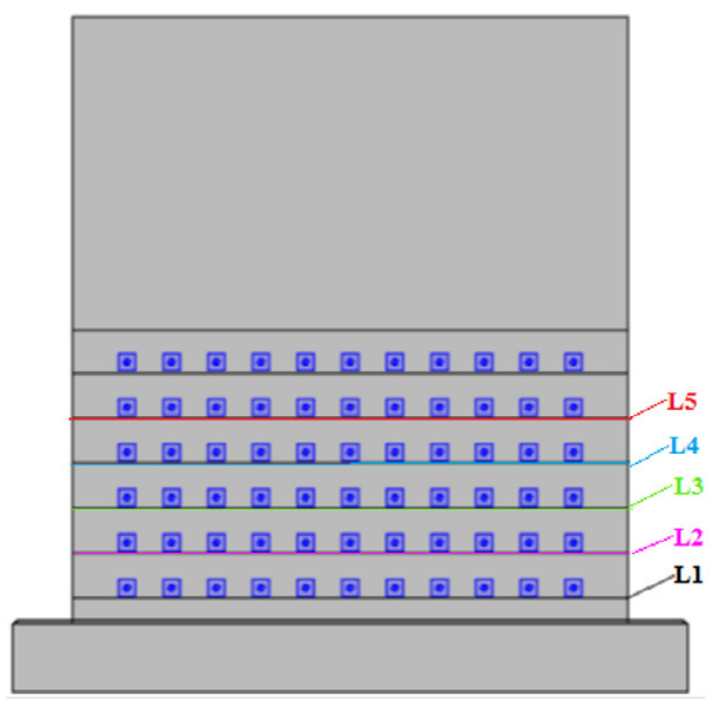

(b)

Fig. 7. (a) Experimental prototype of the LR plate with $11 \times 6$ resonators $(a=40 \mathrm{~mm})$. (b) Schematic of scanning area and detection lines.

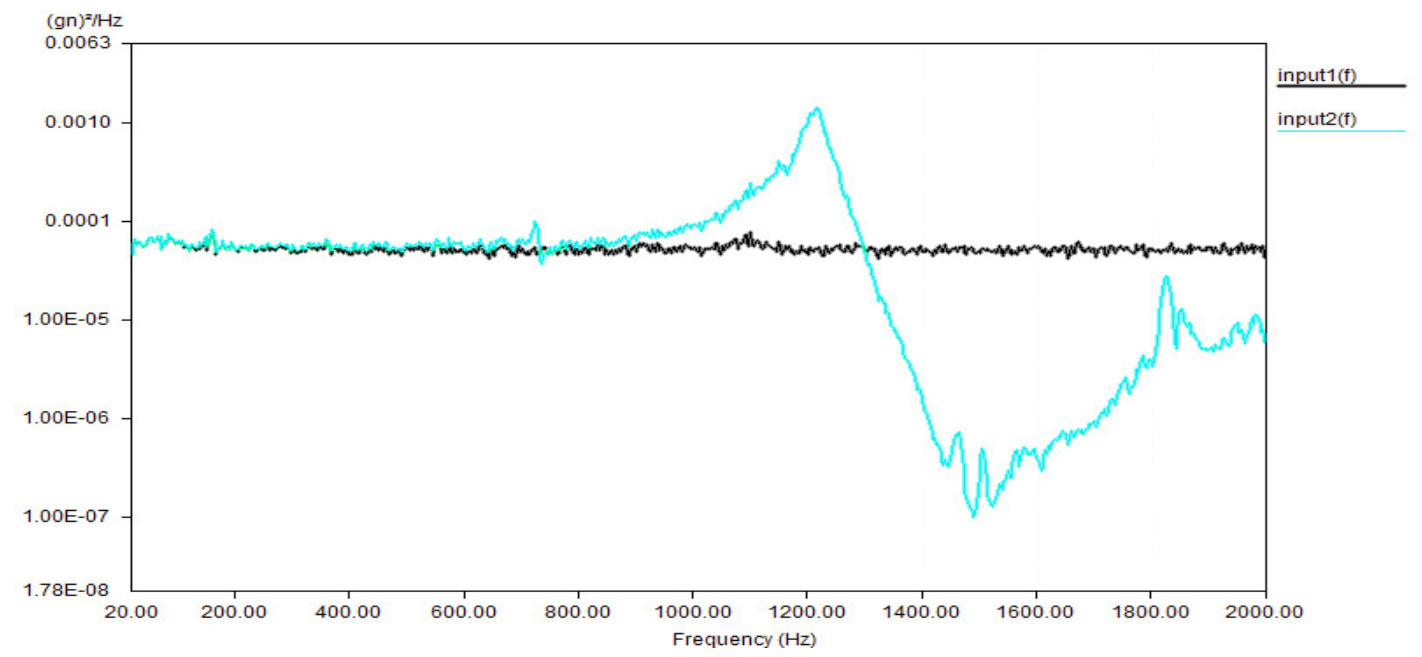

Fig. 8. Measured excitation acceleration of the shaking table (input1) and of the rigid beam (input2).

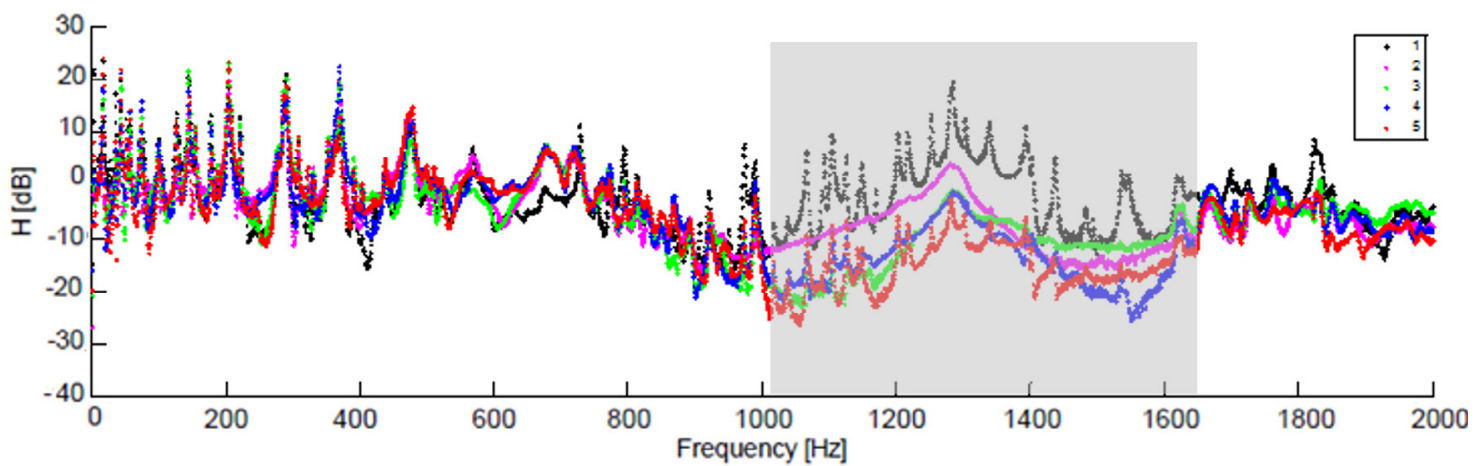

Fig. 9. Measured averaged frequency response $H_{(\text {line(j)) }}$ for the LR plate with periodic resonators $(a=40 \mathrm{~mm})$. 


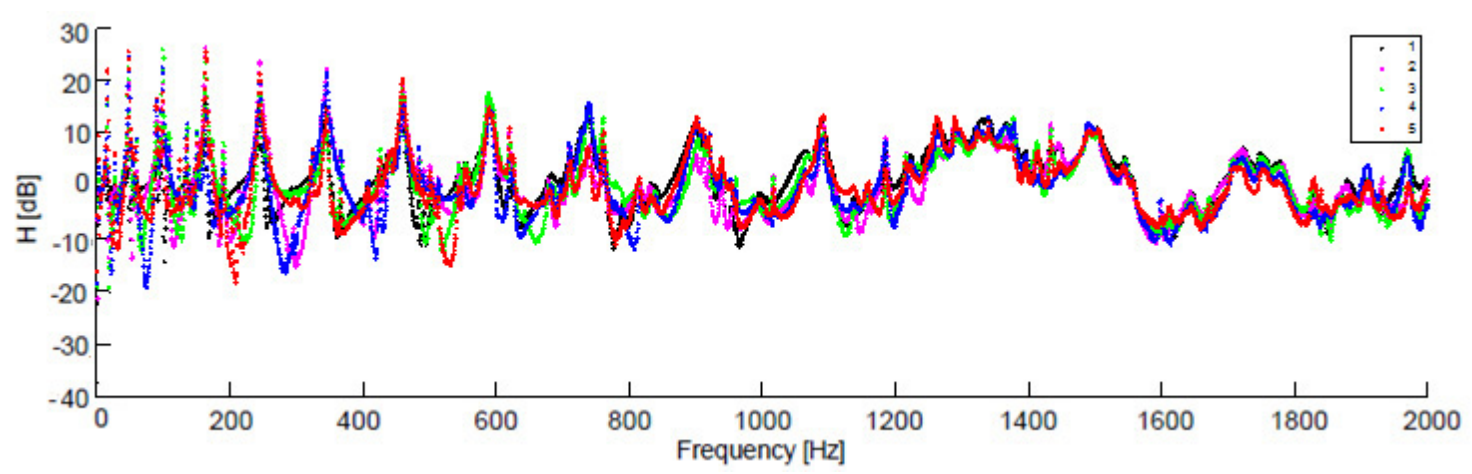

Fig. 10. Measured averaged frequency response $H_{(\text {line(j)) }}$ for the bare aluminum plate.

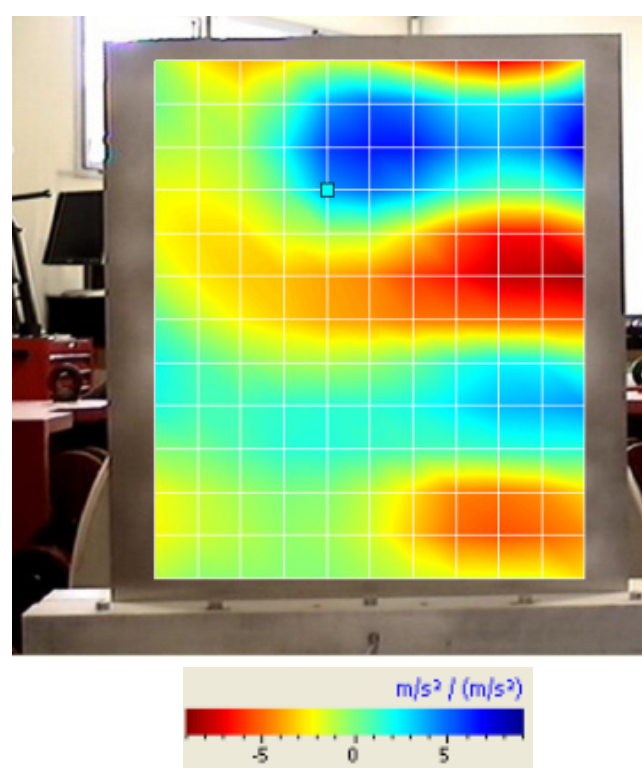

(a)

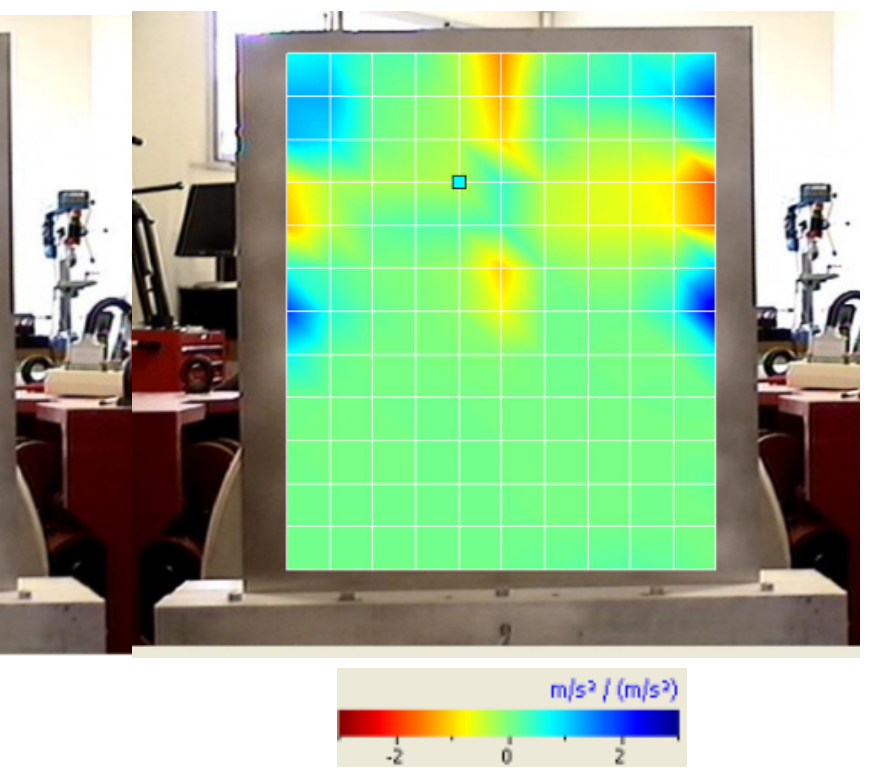

(b)

Fig. 11. Measured vibration shape at frequencies of (a) $f=146 \mathrm{~Hz}$ and (b) $f=1252 \mathrm{~Hz}$.

the existence of a band gap in a frequency range of 1000 to $1650 \mathrm{~Hz}$. This finding is coherent with the theoretical complete band gap (Fig. 5). A Bragg band gap remains, however, difficult to detect. As demonstrated theoretically by Xiao. [4], the attenuation performance of the Bragg band gap is less substantial than that of other band gaps. Also, the band structure (Fig. 4c) shows a horizontal curve at $f=888 \mathrm{~Hz}$ that cuts through the first directional band gap and attenuates its effect. The maximum drive frequency of the shaking table was $2200 \mathrm{~Hz}$; therefore, the resonance band gap in the $O x$-direction [2300 to $3400 \mathrm{~Hz}$ ] cannot be excited.

The displacement fields of the LR plate were plotted for a frequency range outside of the band gap (Fig. 11a) and for a frequency range within the band gap (Fig. 11b). The efficiency of the LR plate is illustrated in Figure 11b: the lower area (treated with LR systems) shows negligible movement compared with the upper area (without LR systems).

To determine the partial band gaps, a second LR plate configuration was tested with a lattice constant of $a=50 \mathrm{~mm}$, corresponding to the case illustrated in Figure 6c. This LR plate was made from the same aluminum base plate as the LR plate used in the first configuration, with a square array of $9 \times 8$ mass-screw resonators (as shown in Fig. 12a). Figure 14 shows the band structure of this configuration: a complete band gap $\left(G_{1}\right.$ [842 to $1056 \mathrm{~Hz}])$ and two partial band gaps ( $g_{1}$ [556 to $858 \mathrm{~Hz}]$ and $g_{3}$ [1714 to $\left.\left.2415 \mathrm{~Hz}\right]\right)$ are visible in the $O x$ direction. For this case, $g_{3}$ wasa resonance gap, and $g_{1}$ was a Bragg gap. Experimentally the unidirectional excitation was more likely to produce plane waves propagating in the $O x$-direction. In Figure 14, the curves of the averaged $H$ values are shown according to frequency for the first six lines of the LR plate depicted in Figure $12 \mathrm{~b}$. Compared with the theoretical frequency gaps (Fig. 13), $H_{\text {line }(j)}$ decreased significantly in the frequency range of the complete band gap $G_{1}$ and the resonance band gap $g_{3}$. Additionally, wave attenuation was low in the frequency range of the Bragg band gap $g_{1}$ (Fig. 14). Nevertheless, this band gap remains difficult to detect, possibly because damping was not taken into account in 


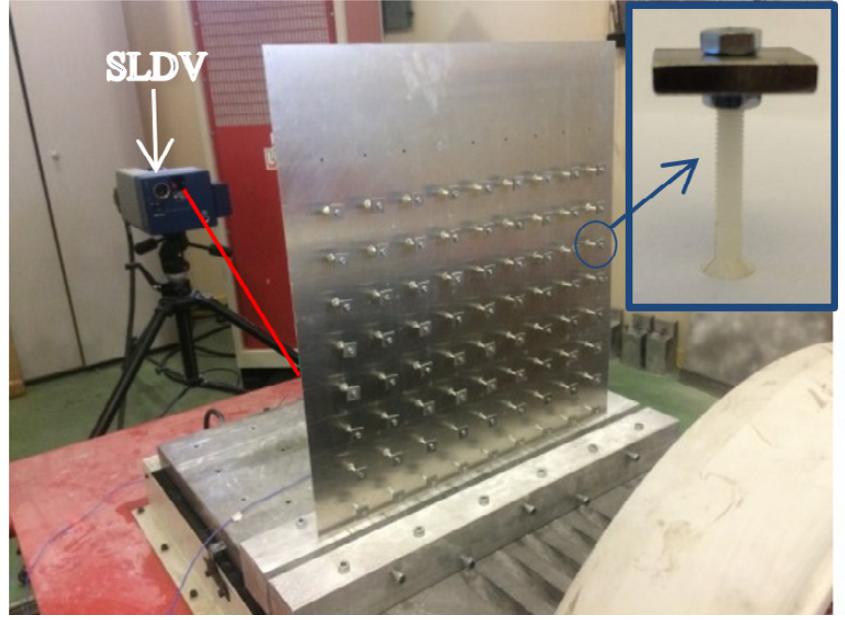

(a)

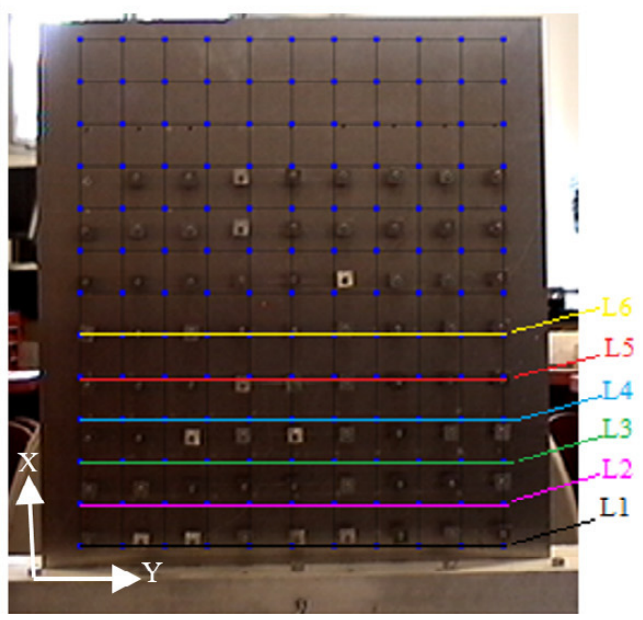

(b)

Fig. 12. (a) Experimental prototype of the LR plate with $9 \times 8$ resonators $(a=50 \mathrm{~mm})$. (b) Schematic of scanning area and detection lines.

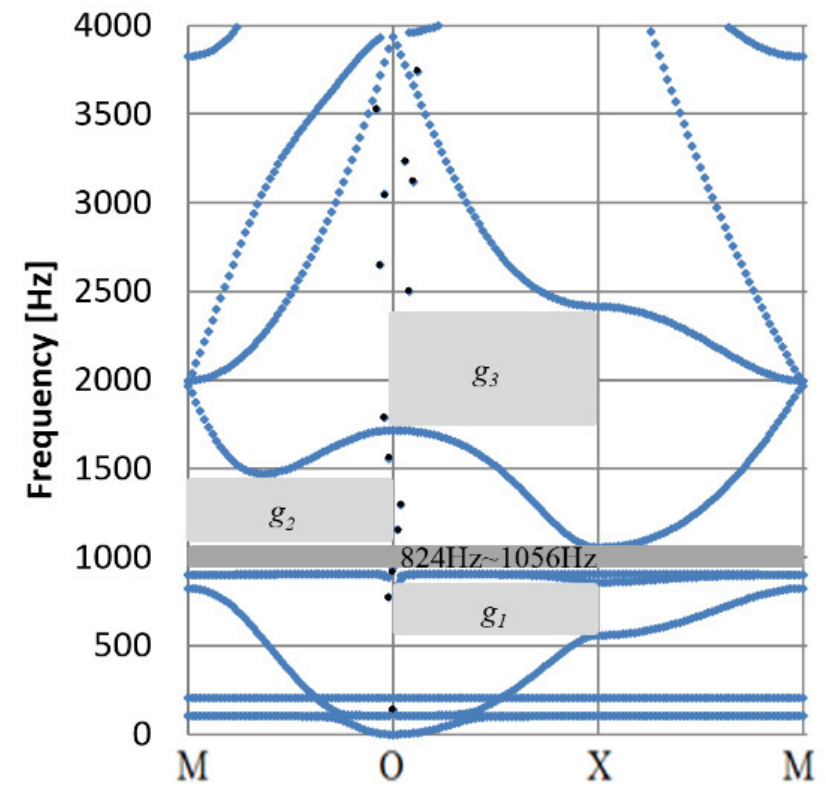

Fig. 13. Band structures of the LR aluminum plate with periodic resonators $(\mathrm{M} 3 \times 25$ screw with top-mounted mass) with a lattice constant of $a=50 \mathrm{~mm}$.

the numerical models (although it was, of course, present in the experiment). According to Peng [12] and Claeys [2], the addition of damping widens the attenuation region but decreases the peak attenuation until the band gap effect is destroyed.

Contrary to the band gap predicted based on an analysis of a unit cell with periodic Bloch-Floquet boundary conditions, experimental band gaps could be affected by boundary reflections. Two types of reflection exist:

- Boundaries in the lateral direction: Edge effects only play a role if waves propagate in a direction other than $x$. In fact, the unidirectional excitation mode mini- mizes the amplitude of these inclined waves. Thus, finiteness of the plate in the $y$-direction should not influence the results. Nevertheless, using punctual excitation results in higher edge-side disturbances, which render the detection of a directional band gap more difficult.

- Boundaries in the $x$-direction: The uniform acceleration field in the untreated area normally extends in all directions and, given the free boundary conditions at the edge of normal $x$, offers acceptable solutions. Thus, the wave propagates mostly in the $x$-direction.

\subsection{Conclusion}

The flexural wave propagation and vibration attenuation characteristics of a LR plate were investigated theoretically and experimentally with a $2 \mathrm{D}$ periodic array of attached resonators (mass-screws). The band gap behavior was characterized theoretically by plotting the band structures of various resonator configurations; a mass was mounted to either the top or middle of a nylon screw to vary the resonator stiffness. In addition, the effect of the lattice constant on the band gap was investigated. The larger the lattice constant $a$, the lower the upper edge of the band gap and the thinner the band gap. Furthermore, combining the resonance gap with the Bragg gap increased the width of the complete band gap. Finally, different lattice constants $(a=40$ and $50 \mathrm{~mm}$ ) were used to obtain two experimental measurements for an LR plate consisting of a periodic array of resonators (mass-screws) attached to a thin homogeneous plate. The theoretical predictions for the position and width of the complete band gap and of the directional band gap are in agreement with the experimental measurements. Compared with a Bragg gap, a much higher attenuation performance was observed for the resonance gap. The Bragg gap was difficult to detect experimentally. 


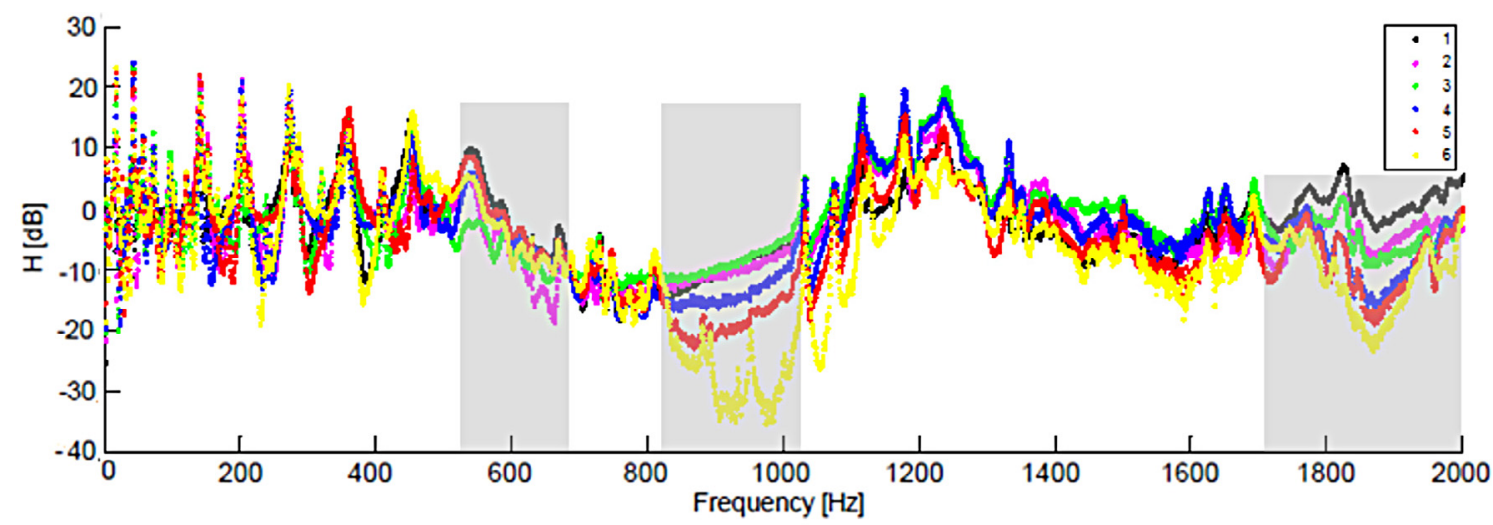

Fig. 14. Measured averaged frequency response $H_{(\text {line }(\mathrm{j}))}$ for the LR plate with periodic resonators $(a=50 \mathrm{~mm})$.

\section{References}

[1] L. Brillouin, Wave propagation in periodic structures Brillouin, 1946

[2] C.C. Claeys, K. Vergote, P. Sas, W. Desmet, On the potential of tuned resonators to obtain low-frequency vibrational stop bands in periodic panels, J. Sound Vib. 332 (2013) 1418-1436

[3] Z. Liu, X. Zhang, Y. Mao, Y.Y. Zhu, Z. Yang, C.T. Chan, P. Sheng, Locally Resonant Sonic Materials, Science 289 (2000) 1734-1736

[4] Y. Xiao, J. Wen, X. Wen, Flexural wave band gaps in locally resonant thin plates with periodically attached spring-mass resonators, J. Phys. Appl. Phys. 45 (2012) 195401

[5] M. Oudich, M. Senesi, M.B. Assouar, M. Ruzenne, J.H. Sun, B. Vincent, Z. Hou, T.-T. Wu, Experimental evidence of locally resonant sonic band gap in twodimensional phononic stubbed plates, Phys. Rev. B 84 (2011) 165136

[6] K. Yu, T. Chen, X. Wang, Band gaps in the low-frequency range based on the two-dimensional phononic crystal plates composed of rubber matrix with periodic steel stubs, Phys. B Condens. Matter 416 (2013) 12-16
[7] Y. Li, T. Chen, X. Wang, Y. Xi, et Q. Liang, Enlargement of locally resonant sonic band gap by using composite plate-type acoustic metamaterial, Phys. Lett. A 379 (2015) 412-416

[8] A. Hu, X. Zhang, F. Wu, Y. Yao, Enlargement of the locally resonant Lamb wave band gap of the phononic crystal plate at the deep sub-wavelength scale, Mater. Res. Express 1 (2014) 45801

[9] M. Nouh, O. Aldraihem, A. Baz, Wave propagation in metamaterial plates with periodic local resonances, J. Sound Vib. 341 (2015) 53-73

[10] M. Oudich, Y. Li, B.M. Assouar, Z. Hou, A sonic band gap based on the locally resonant phononic plates with stubs, New J. Phys. 12 (2010) 83049

[11] P.Y. Chen, C.G. Poulton, A.A. Asatryan, M.J. Steel, L.C. Botten, C. Martijn de Sterke, R.C. McPhedran, Folded bands in metamaterial photonic crystals, New J. Phys. 13 (2011) 53007

[12] H. Peng, P. Frank Pai, Acoustic metamaterial plates for elastic wave absorption and structural vibration suppression, Int. J. Mech. Sci. 89 (2014) 350-361 